\title{
RF-KICKER SYSTEM FOR SECONDARY BEAMS AT THE NSCL
}

\author{
D. Gorelov, V. Andreev, D. Bazin, M. Doleans, T. Grimm, F. Marti, J. Vincent, X. Wu \\ NSCL/MSU, East Lansing, MI 48824, U.S.A.
}

\begin{abstract}
The design and construction of a radio frequency fragment separator (RFFS) kicker system at the National Superconducting Cyclotron Laboratory (NSCL) of Michigan State University (MSU) has been proposed. This RFFS will be used to further purify secondary beams of rare isotopes after the exiting the A1900 Fragment Separator and will open a wide range of possibilities for new experiments at the forefront of nuclear science. The proposed system is studied as an efficient alternative to the traditional approach using a Wien filter. Rare neutron deficient secondary beams are challenging to purify because of the presence of intense contaminants that cannot be removed by the traditional energy loss method. However, velocity differences resulting in time-of-flight (TOF) differences can be used for the effective separation of the beams transversely using the time-varying electromagnetic fields of the RF kicker. Its technical design is presented together with the beam dynamics analysis of a secondary beam in realistic 3D electromagnetic fields. The expected purification improvement of the exotic beams for the foreseen nuclear physics experiments is shown in detail.
\end{abstract}

\section{INTRODUCTION}

We propose the construction of the RFFS system to purify beams of rare neutron deficient isotopes. This will open up the possibility of a wide range of new experiments at the forefront of nuclear science and is essential to achieve some of the important science goals of the NSCL and the nuclear physics community at large regarding the exploration of extremely neutron deficient nuclei.

The NSCL Coupled Cyclotron Facility (CCF) produces some of the most intense beams of neutron deficient nuclei such as ${ }^{100} \mathrm{Sn}$ available world wide. Previous attempts to perform experiments with these neutrondeficient beams met with serious challenges due to large contaminations and overpowering background from other undesired ions that cannot be separated with the existing A1900 fragment separator. The RF fragment separator proposed here will add another criterion to the selection of rare isotopes and solve this problem by rejecting unwanted particles according to their velocities. The enhanced signal to background ratio for the typically weak rare isotope beams translates directly into enhancements in discovery potential and at least a factor of ten in efficiency for the many experiments that are limited by trigger detectors being overwhelmed with background. These experiments will address a wide range of important science ranging from astrophysical X-ray bursts, over nuclear structure to experiments relating to precision tests of the Standard Model.

\section{PROJECT DESCRIPTION}

The central device in the RFFS is an RF kicker that is operated at the cyclotron frequency with an adjustable phase difference. The RF transverse electric field causes a phase dependent transverse deflection of the beam. Ions in the beam that differ in velocity and therefore in timeof-flight will arrive at the RF kicker with different phases relative to the kicker frequency and will therefore experience different transverse angular deflections. After a few meters of drift this will result in a transverse separation and the desired ion can be selected with a slit system while rejecting unwanted contaminants providing an effective velocity filter. As different ion species experience different field strengths much weaker fields can be used compared to a Wien filter to obtain sufficient spatial separation. Such an approach has also been chosen by RIKEN where a similar device is in use [1].

\section{Conceptual Design}

Fig. 1 shows the schematic diagram of the RFFS, which will be occupying the S1 experimental hall located after the NSCL experimental area main fragment separator A1900.

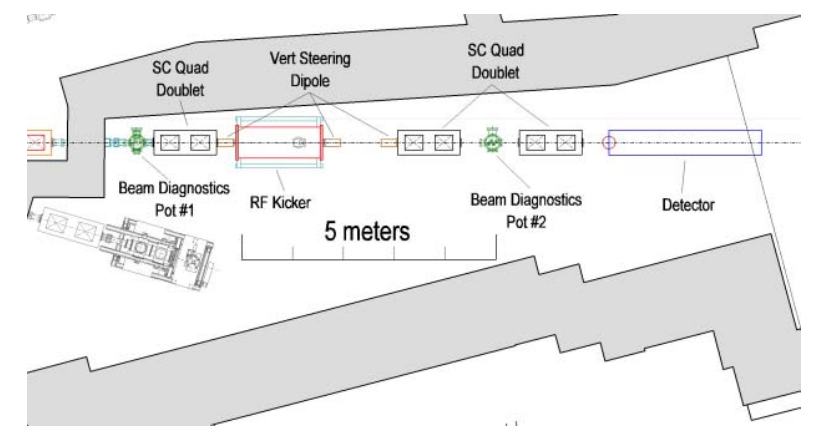

Figure 1. Schematic diagram of the proposed RFFS.

The existing beam line will transport the secondary beams from the A1900 extended focal plane to the first beam diagnostics box with the desired transverse beam conditions. The focusing elements located between the last dipole of the beam line and the wall of the S1 experimental hall will be used for the transverse phase space matching. The first quadrupole doublet of the RFFS will provide additional transverse focusing for the secondary beam at the entrance of the RF kicker that will produce a vertical beam separation for different secondary beam species according to the difference in their time-offlight from the A1900 production target. Three vertical steering dipole magnets before and after the RF kicker will be used to cancel the kicker's effect on the $b$ eam centroid of the desired ion and keep it on the beam axis. The second quadrupole doublet will achieve the desired 
beam transverse phase space at the slit inside the second beam diagnostics pot. After the desired secondary beam is selected with the selection slit, the third quadrupole doublet will provide the transverse focusing to the experimental setup. These three quadrupole doublets will also provide sufficient flexibility in the transverse matching to support other possible experiments that do not require the RFFS.

The RF kicker will be operated at the K1200 superconducting cyclotron frequency in the range of $21 \div 28 \mathrm{MHz}$, depending on the primary beam energy required for the experiment. The proposed RF kicker design uses a pair of $1.5 \mathrm{~m}$ long parallel plates, with a width of $10 \mathrm{~cm}$ and gap of $5 \mathrm{~cm}$. The design accomodates secondary beams with transverse emittances of about $80 \pi \mathrm{mm}$ mrad. The electric field uniformity within the beam region is about $+/-2 \%$. The voltage applied on these plates will be sinusoidal with a peak voltage of $100 \mathrm{kV}$, resulting in a maximum electric field of $20 \mathrm{kV} / \mathrm{cm}$.

\section{Performance}

We have simulated the utilization of the RFFS in various types of experiments. One of the most important experiments to be performed with the RFFS is the $\beta$ decay of ${ }^{100} \mathrm{Sn}$. The goal is to obtain sufficient beam purity to allow the correlation of decays with implants, to keep backgrounds low, and at the same time to avoid the loss of the rare ${ }^{100} \mathrm{Sn}$ ions. To achieve these goals a final beam intensity of less than a few ions per second including all contaminants is necessary. With an estimated production rate for ${ }^{100} \mathrm{Sn}$ of $6 \times 10^{-4}$ per second, this requires a beam purity (desired fragment intensity per total intensity) of about $2 \times 10^{-4}$.

The production of the secondary beam species including ${ }^{100} \mathrm{Sn}$ was calculated with the code LISE $[2,3]$ assuming a $140 \mathrm{MeV} / \mathrm{u}{ }^{124} \mathrm{Xe}$ primary beam from the K1200 cyclotron impinging on a beryllium production target. In this case, the RF frequency of the K1200 cyclotron and of the RF kicker is $\sim 23.1 \mathrm{MHz}$. The A1900 is assumed to operate with a momentum acceptance of $1 \%$ for the adequate limitation of the contaminants intensities and the secondary beam energy after the A1900 is $\sim 90 \mathrm{MeV} / \mathrm{u}$. Those parameters are currently achievable with the A1900 and were chosen to obtain good separation in RF phase between the different secondary beams at the kicker location. The LISE and TRANSPORT codes were used to estimate the secondary beam transverse phase space parameters, TOF and intensity distributions at the entrance of RFFS about $52 \mathrm{~m}$ downstream from the A1900 production target. The acceptance of the existing beam line is assumed to be $<80 \pi \mathrm{mm}$ mrad. Benchmarking between the results from LISE and the available experimental data from test experiments had been done and gives confidence in the capability to reasonably reproduce the difference in the intensity of the contaminant beams with respect to the ${ }^{100} \mathrm{Sn}$ beam using LISE.

For the case considered, the purity of the ${ }^{100} \mathrm{Sn}$ beam out of A1900 when compared to the total beam intensity together with the contaminants is $\sim 10^{-6}$. The left panel of Fig. 2 illustrates the contamination problem in this case. However if the proposed RFFS system is used, the vast majority of the contaminants can be effectively removed as shown in Fig. 2 on the right

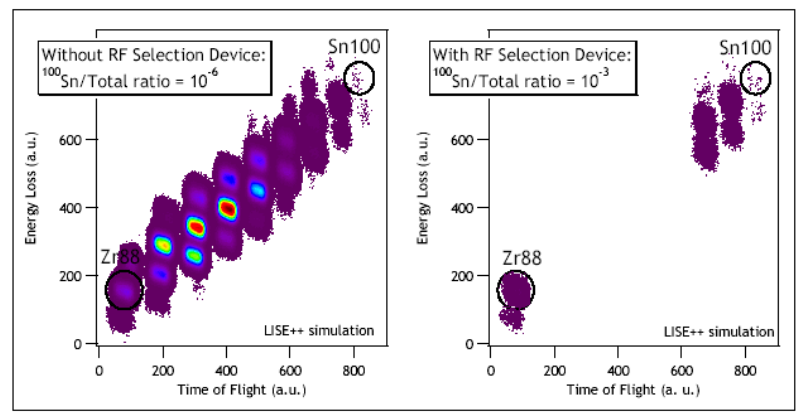

Figure 2: Simulated energy loss in a detector versus the time of flight used for the particle identification without and with the RFFS for a ${ }^{100} \mathrm{Sn}$ experiment.
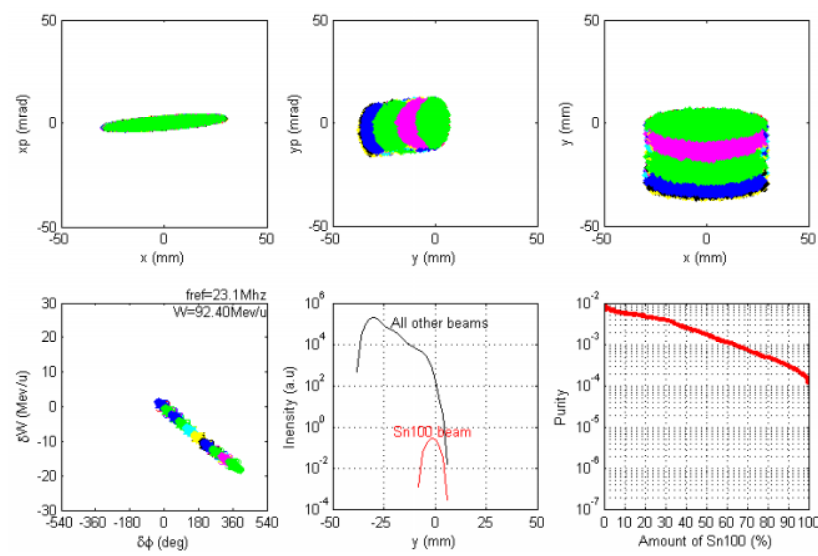

Figure 3: Top row, left to right $-x-x^{\prime}, y-y^{\prime}, x-y$ phase space projections. Bottom row, left to right - longitudinal phase space projection, vertical beam profile, and the dependance of the ratio of the ${ }^{100} \mathrm{Sn}$ beam and the total beam intensities (purity) as a function of the ${ }^{100} \mathrm{Sn}$ ions fraction accepted at the final selection slit compared to its intensity at the entrance to the RFFS (transmission).

The beam dynamics simulation results with the ideal transverse single harmonic electric field are shown in Fig. 3. The edge effects of the electromagnetic fields in the RF kicker were investigated using the code LANA [4] with the 3D beam dynamics in realistic fields calculated with MAFIA. The fringe fields and the nonuniformity of the transverse electric field across the aperture in the resonator were found to have no significant effect on the beam quality. As shown in Fig. 3 the desired purity of $2 \times 10^{-4}$ can be achieved for the transmission of more than $90 \%$ of the ${ }^{100} \mathrm{Sn}$ ions.

A different experiment to study the ${ }^{21} \mathrm{Mg}$ magnetic moment measurements needs a significant suppression of the ${ }^{20} \mathrm{Na}$ contaminant. Similar analysis was performed for this case as well. The simulations predict that the RFFS can be used to reduce the contamination in this case by 5 orders of magnitude without affecting noticeably the intensity of the ${ }^{21} \mathrm{Mg}$ beam. 


\section{Hardware}

The required hardware for the proposed RFFS is one RF kicker, three SC quadrupole doublets, three steering dipole magnets and two beam diagnostic pots.

Fig. 4 shows the RF kicker design with capacitive tuners on the top and bottom, and RF power coupler on the side of the RF kicker vacuum chamber. A Splitcoaxial type resonant structure has been chosen to create the RF voltage between the electrodes (deflecting plates). The structure is rather simple to manufacture and has a high quality factor and acceptable RF power losses. To simulate the resonator the code MAFIA (v. 4.106) was used. Two capacitive tuners will provide a resonant frequency change of the RF kicker in the range of $21 \div 28 \mathrm{MHz}$. In this case, the distance between the tuner plates and the electrodes will be changed from 20 to 110 $\mathrm{mm}$. A movable power coupler will be able to excite the resonator within the total range of the frequencies, providing a reasonable voltage standing wave ratio (VSWR) in the RF power line. Two RF pickups have been foreseen for the control system. The main RF parameters of the resonator are shown in Table 1. As can be seen the quality factor $(\mathrm{Q})$ and the total RF power losses of the resonator $\left(\mathrm{P}_{\mathrm{tot}}\right)$ depend on the resonant frequency. The higher the RF frequency, the higher the $\mathrm{Q}$ and the lower the power losses. A water-cooling system will be used to cool the resonator. The stiffness of the structure will be improved if necessary by installing additional reinforcement ribs at the electrodes.

Three quadrupole doublets will be required to provide the transverse focusing for the secondary beam in the RFFS. Three existing NSCL superconducting beam line quadrupole doublets will be adequate. The quadrupoles in all three doublets have an aperture of $6.35 \mathrm{~cm}$, effective length of $35.6 \mathrm{~cm}$ and a maximum magnetic gradient of $35 \mathrm{~T} / \mathrm{m}$.

Three vertical steering dipole magnets will be required to position the desired secondary beam on axis after the RF kicker in the RFFS. The magnets with an effective length of $30 \mathrm{~cm}$ and an aperture of $10 \mathrm{~cm}$ are identical to the existing prototypes at the NSCL. The transverse magnetic field requirement of $0.05 \mathrm{~T}$ is easily achievable.

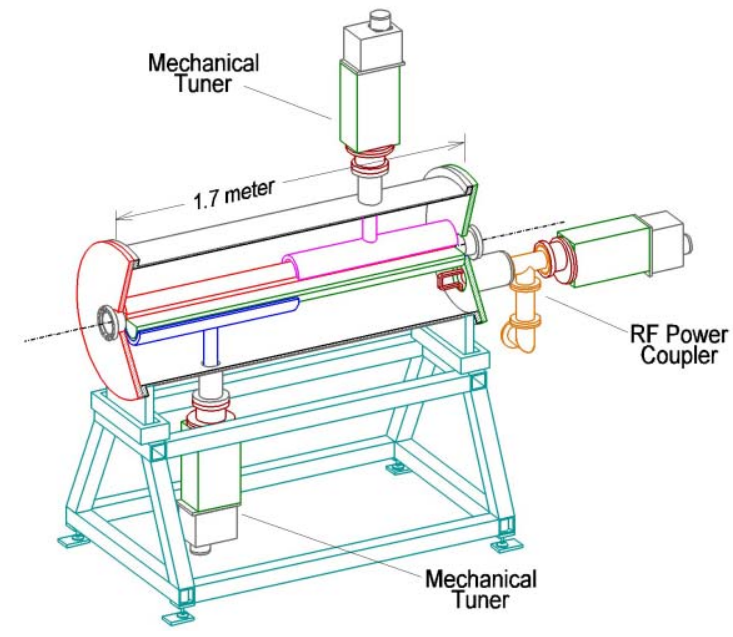

Figure 4: RF kicker structure.

\section{CONCLUSION}

The RFFS system project was proposed as an efficient upgrade of the CCF at the NSCL at Michigan State University. The future plans for the design and construction of this project are in the finalization stage at the laboratory. The possible funding from NSF this summer can make this development possible in the next few years.

\section{REFERENCES}

[1] K. Yamada, T. Motobayashi, and I. Tanihata, " $R F$ deflector system for proton-rich RI beams in RIKEN", Nucl. Phys. A 746 (2004) 156Y.

[2] D. Bazin, O. Tarasov, M. Lewitowicz, and O. Sorlin, "The program LISE: a simulation of fragment separators", Nucl. Instr. and Methods in Phys. Research A482 (2002), 307.

[3] O. Tarasov, "Analysis of momentum distributions of projectile fragmentation products", Nucl. Phys. A734 (2004) 536.

[4] D.V.Gorelov and P.N.Ostroumov, "Applications of the LANA Code for Design of Ion Linacs", Proc. of the LINAC'96 Conf., Sitges, Spain, (1996) p.1271.

Table 1: Main RF parameters for the RF kicker.

\begin{tabular}{|c|c|c|c|c|c|c|c|}
\hline \multirow{2}{*}{$\begin{array}{l}\text { Inter-electrode } \\
\text { voltage }(\mathrm{kV})\end{array}$} & \multirow{2}{*}{$\begin{array}{c}\text { Tuner } \\
\text { distance }(\mathrm{cm})\end{array}$} & \multirow{2}{*}{$\begin{array}{l}\text { Resonant } \\
\text { frequency }(\mathrm{MHz})\end{array}$} & \multirow{2}{*}{$\begin{array}{c}\text { Resonator } \\
\text { quality factor } \\
\text { Q }\end{array}$} & \multicolumn{4}{|c|}{ Power losses } \\
\hline & & & & $\begin{array}{c}\text { Total } \\
\mathrm{P}_{\text {tot }}(\mathrm{kW}) \\
\end{array}$ & $\begin{array}{c}\text { Electrodes } \\
\mathrm{P}_{\mathrm{pl}}(\mathrm{kW})\end{array}$ & $\begin{array}{c}\text { Tuner } \\
\mathrm{P}_{\text {tun }}(\mathrm{kW})\end{array}$ & $\begin{array}{c}\text { Cavity } \\
\mathrm{P}_{\text {cav }}(\mathrm{kW}) \\
\end{array}$ \\
\hline 100 & 2 & 21.240 & 9540 & 10.15 & 5.02 & 3.05 & 2.08 \\
\hline 100 & 3 & 22.960 & 10100 & 8.5 & 4.5 & 2.2 & 1.8 \\
\hline 100 & 4 & 24.360 & 10700 & 7.4 & 4.1 & 1.8 & 1.5 \\
\hline 100 & 5 & 25.430 & 11130 & 6.6 & 3.8 & 1.4 & 1.4 \\
\hline 100 & 7 & 26.932 & 11900 & 5.7 & 3.45 & 0.95 & 1.3 \\
\hline 100 & 9 & 27.862 & 12400 & 5.15 & 3.25 & 0.7 & 1.2 \\
\hline 100 & 11 & 28.350 & 12400 & 5.1 & 3.2 & 0.6 & 1.3 \\
\hline
\end{tabular}

